\title{
Capacity credit of microgeneration and microgrids
}

\author{
Paulo Moisés Costa ${ }^{\mathrm{a}, \mathrm{c}, *}$, Manuel A. Matos ${ }^{\mathrm{b}}$ \\ a Escola Superior Tecnologia e Gestão de Viseu, Instituto Politécnico Viseu, Viseu, Portugal \\ ${ }^{\mathrm{b}}$ INESC Porto, Faculdade de Engenharia, Universidade do Porto, Porto, Portugal \\ ${ }^{\mathrm{c}}$ INESC Porto, Portugal
}

\section{A R T I C L E I N F O}

\section{Article history:}

Received 6 January 2010

Accepted 14 June 2010

Available online 3 July 2010

\section{Keywords:}

Microgeneration

Microgrid

Capacity credit

\begin{abstract}
A B S T R A C T
The recent development of the concept of microgrid ( $\mu \mathrm{Grid}$ ), associated to the emergent interest in microgeneration $(\mu \mathrm{Gen})$, has raised a number of challenges regarding the evaluation of the technical, economical and regulatory impacts of a high penetration of this kind of solutions in the power systems. In this paper, the topic of security of supply is addressed, aiming at evaluating the influence of $\mu$ Gen and $\mu \mathrm{Grids}$ in the medium- and long-term availability of generation to serve the forecasted load. A MonteCarlo based methodology is used to evaluate this influence and to assess the capacity credit of those entities.
\end{abstract}

(c) 2010 Elsevier Ltd. All rights reserved.

\section{Introduction}

The integration of distributed generation (DG) on power systems has been increasing for the last decades, mainly in MV and HV levels (due to technical, safety and economical reasons). However, in the last years, the interest on microgeneration systems ( $\mu \mathrm{Gen}$ ) connected to LV networks has increased as well, essentially due to the technological development of those generators, the decline of their costs and the public incentives to DG. Moreover, the concept of microgrid ( $\mu \mathrm{Grid}$ ) has been studied as a way to ease the integration of $\mu \mathrm{Gen}$ in LV networks (Saraiva et al., 2003; DTI, 2004; Caire et al., 2002; Project $\mu$ grids, 2008; Pudjianto et al., 2005). A $\mu$ Grid is an association of a LV distribution network, microgenerators, loads and storage devices, having some local coordinated functions (Costa and Matos, 2009; Lasseter and Piagi, 2004). This entity can operate interconnected with the distribution network or isolated from it (using local resources).

The development of the concept of $\mu \mathrm{Grid}$, associated to the emergent interest in $\mu \mathrm{Gen}$, raised a number of challenges regarding the evaluation of the technical, economical and regulatory impacts of a high penetration of this kind of solutions in the power systems. In a previous paper of the authors (Costa et al., 2008), a regulatory framework for the economic integration of $\mu \mathrm{Gen}$ and $\mu \mathrm{Grid}$ on distribution networks was presented. The proposed framework seeks to remove potential barriers to $\mu \mathrm{Gen}$ and $\mu$ Grids development namely defining an incentive mechanism to those entities. This mechanism is funded by the avoided

\footnotetext{
*Corresponding author at: Escola Superior Tecnologia e Gestão de Viseu, Instituto Politécnico Viseu, Viseu, Portugal. Tel.: +351232480500; fax: +351232424651 .

E-mail address: Paulomoises@elect.estv.ipv.pt (P. Moisés Costa).
}

costs those entities may produce, including the ones related to the security of supply.

In this paper, the detailed model of the Monte-Carlo based methodology used to assess the capacity credit of $\mu \mathrm{Gen}$ and $\mu \mathrm{Grids}$ is presented. It is worth noting that some of the values of CC found by other authors are not comparable to the ones calculated in this paper, since they were not obtained for microgenerators. This is especially true for wind power.

The paper is organized as follows: In Section 2, the concept of capacity credit is presented. Then, in Section 3, a methodology to assess the capacity credit of $\mu \mathrm{Gen}$ and $\mu \mathrm{Grid}$ is proposed. An illustrative example is included in Section 4. The conclusions and references complete the paper.

\section{Capacity credit}

In broad terms, the concept of capacity credit (CC) is a measure of how a specific entity contributes to the generating adequacy of a power system (i.e., the availability of generation capacity to serve the forecasted load-Hierarchical Level 1 (HL1) reliability study). However, there is not a single definition for this concept. In fact, the formal definition of CC and the methodologies used to estimate its value vary widely in different sources. The more simplistic approaches use "rule-of-thumb" models (Pudaruth and Li, 2009; Bae and Kim, 2007; Garver, 1966), where the CC of a specific entity is calculated without considering its impact on the system reliability. For instance, the $\mathrm{CC}$ of a wind farm may be defined as the fraction of the installed wind capacity that either is online at all times or with a probability similar to the availability of a fossil fuel power plant (Pudaruth and Li, 2009). Those approaches do not account for the stochastic and variable nature 
of some resources (for instance, the wind velocity). Nevertheless, the majority of methods proposed to evaluate the CC are based on probabilistic approaches, which account for the stochastic nature of the intermittent generation. Such methods involve the development of appropriate generation and load models and their combination to create a risk model containing the required adequacy indices. Several indices may be used to evaluate the generating adequacy of a power system. The most commonly used are: the loss of load probability (LOLP); the loss of load expectation (LOLE-h/yr); the expected power not supplied (EPNS-MW); and the expected energy not supplied (EENS-MWh).

A number of CC definitions to be used with probabilistic methods have been proposed, namely: the equivalent firm capacity (EFC); the load carrying capability (LCC); the equivalent conventional power plant (ECC); and the guaranteed capacity (GC). Some EFC and ECC approaches define the CC as the amount by which the firm (thermal) capacity of the system can be decreased, after the addition of the capacity whose CC is to be determined, while maintaining the same reliability (Pudaruth and Li, 2009; Hawkes and Leach, 2008; He Yau, 2006). The difference between EFC and ECC has to do with the nature of the reference generator. EFC methods use a 100\% reliable unit, whereas ECC approaches consider a "conventional" unit, with a specific forced outage rate. This ECC concept is used in this paper to assess the CC of $\mu$ Gen and $\mu$ Grids. Nevertheless, some authors define the CC as the capacity of a reference generator that should be added to the system in order to obtain the same reliability level as the one resulting from the addition of the capacity whose CC is to be determined (Amelin, 2009; Milligan and Porter, 2006).

Regarding LCC, the CC is defined as the amount by which load may be increased (usually a constant value), after adding the capacity whose CC is to be determined, while the original reliability of the system is maintained (Pudaruth and Li, 2009; Amelin, 2009; Milligan and Porter, 2006; Luickx et al., 2009). Finally, the GC concept defines the CC without taking into consideration the load of the system (Amelin, 2009, Deutsche Energie-Agentur GmbH, 2005). In this case, the CC is defined as the difference between the "guaranteed capacity" that exists with and without the capacity whose CC is to be determined. In this context, the concept of "guaranteed capacity" corresponds to the capacity level that is expected to be available with a given probability $\rho$ (e.g. $\rho=99 \%$ (Deutsche Energie-Agentur GmbH, 2005)). It is important to stress that the probability $\rho$ is an arbitrarily chosen parameter that should not be mistaken for the LOLP of the system (Amelin, 2009).

A comparison between the EFC, LCC, ECC and GC approaches was made by Amelin (2009). The results show that the choice of definition can have significant impact on the obtained CC. Moreover, the results show that the EFC and the LCC provide consistent results. As well, it can be concluded that the ECC approach provides CC values that follow the same trends as EFC and LCC, despite the higher values obtained (due to the consideration of a not $100 \%$ reliable reference generator). The results obtained when using GC approach are not correlated to the results of the other three methods. Amelin (2009) found that the CC depends on $\rho$ in an unpredictable manner. Moreover, once the load is not included in the model, the GC approach does not take into consideration the real necessity of the available capacity.

\section{Methodology}

\subsection{Overall process}

The addition of $\mu$ Gen capacity to a power system always improves its generating adequacy, once it increases the available generation. The establishment of $\mu$ Grids permits to increase the contribution of some $\mu \mathrm{Gen}$ systems to the adequacy, namely the one of the micro-CHP units. Actually, those generators may become controllable when integrated into $\mu$ Grids. As a result those systems tend to be always available to respond to capacity scarcity situations. Nevertheless, only micro-CHP systems with ability to store thermal energy should be assumed as controllable. In fact, only those units may respond to the needs of the electrical system without wasting the thermal energy (preserving the principle of combined heat and power generation).

The contribution of the $\mu$ Grids to the generation adequacy also results from the ability of those structures to interrupt part of their internal load when a situation of generation scarcity exists. The interruption may be done, sometimes, without noticeable effects on consumers, reducing the potential economic cost that results from the load interruption. Actually, the $\mu$ Grids may be used to interrupt the less important loads (heat load, cooling, etc.),

The contribution of the $\mu \mathrm{Gen}$ and $\mu$ Grids to the generation adequacy results in a CC to those entities. This CC may be estimated using the procedure presented in Fig. 1, which is based on the ECC concept previously mentioned. Note that, in the algorithm, the loss of load expectation (LOLE) was adopted as the measure of the system reliability. This index was chosen because it is the most commonly used when assessing the CC. However, a different reliability index may be used, namely the loss of load probability (LOLP) and the expected energy not supplied (EENS) (Milligan, 2000; Milligan and Porter, 2006; Pudaruth and Li, 2008; Luickx et al., 2009; Pudaruth and Li, 2009).

As Fig. 1 shows, the CC evaluation implies successive computation of the system's LOLE. A reliability model based on a non-chronological Monte-Carlo simulation (MCS) process may be used for this purpose. This approach helps accounting for the relatively large number of random variables involved, namely

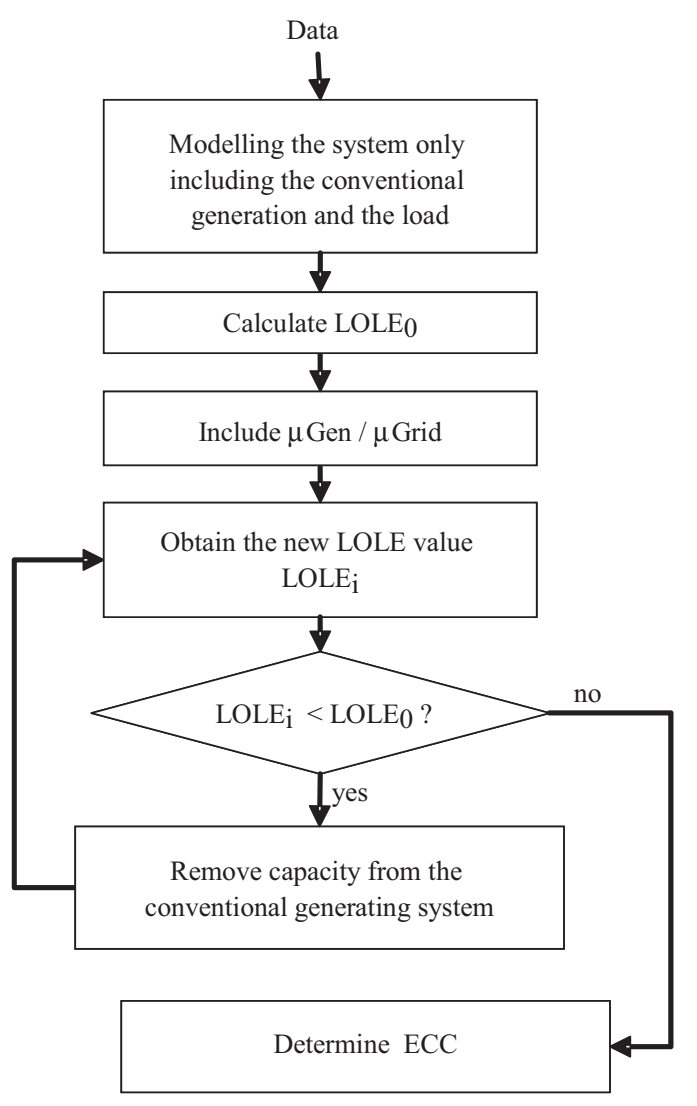

Fig. 1. Flowchart for CC computation. 
related to: (i) the generation profiles of $\mu \mathrm{Gen}$; (ii) the probability of failure of the generators (including the one of microgenerators); (iii) and the system load level.

Fig. 2 shows the algorithm of the MCS methodology, where $N$ is the number of trials; $F$ is the number of loss of load occurrences; and PNS is the power not supplied due to loss of load situations. In broad terms, in every single trial of the MCS process, the available generation and the system load are sampled and compared in order to assess the generation adequacy of the system. This procedure is repeated until a convergence criterion is achieved. In this case, the coefficient of variation of the expected energy not

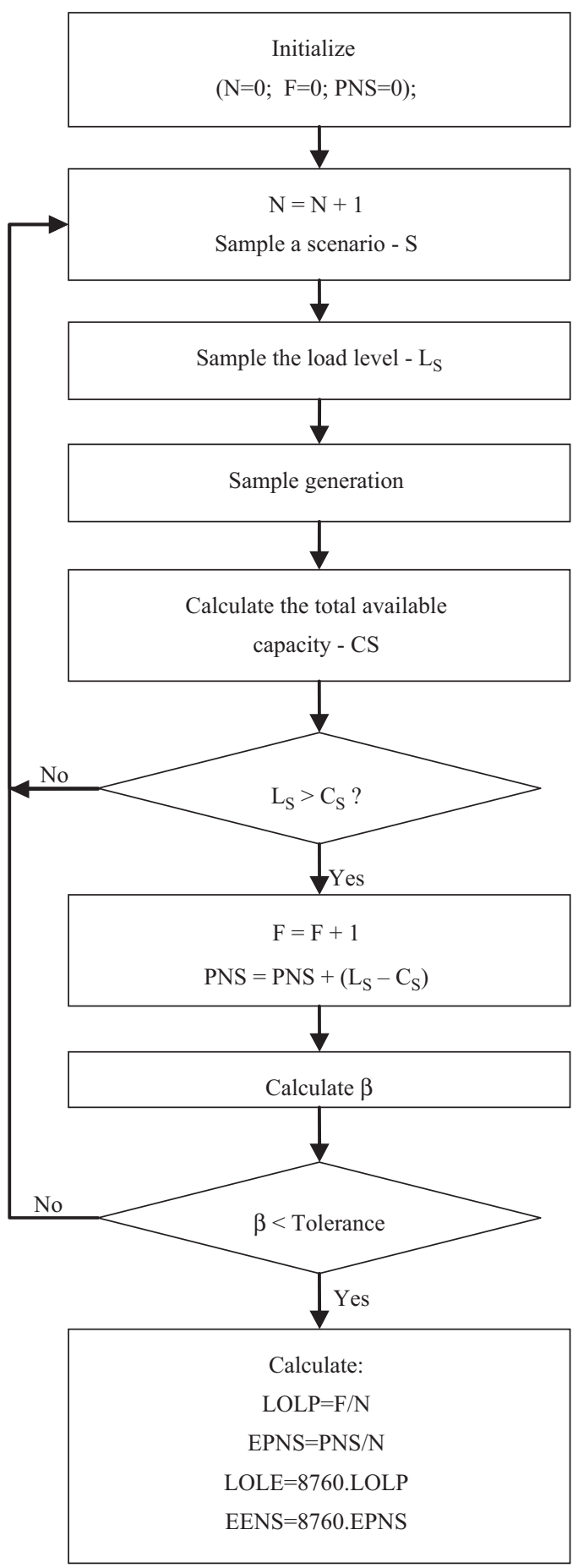

Fig. 2. Algorithm for non-chronological Monte-Carlo with scenarios. supplied index (EENS) was adopted:

$$
\beta=\frac{\frac{\sigma(\mathrm{EENS})}{\sqrt{N}}}{\mathrm{EENS}}
$$

where $\sigma$ (EENS) is the standard deviation of the expected value of the EENS, and EENS is calculated by

$\mathrm{EENS}=8760 \times \frac{\mathrm{PNS}}{N}$

After the convergence of the MCS process, several reliability indices (besides the EENS) may be calculated, namely the loss of load probability (LOLP), the loss of load expectation (LOLE) and the expected power not supplied (EPNS).

The total available generation on each trial of the MCS process has two contributions: (i) centralized generators; (ii) $\mu \mathrm{Gen}$ systems. The second contribution is influenced by natural conditions, namely: the solar radiation for PV systems; the wind velocity for micro-wind turbines; and the ambient temperature for micro-CHP systems. As a result, the contribution of a specific $\mu$ Gen technology for the generating capacity tends to present seasonal and daily variations. The system load also shows well-known seasonal and daily variations. Therefore, potential dependencies between the generation and the load profiles exist. Those dependencies may influence the CC of the $\mu$ Gen technologies. In fact, a technology with a lower annual capacity factor (CF) may have a bigger contribution to the generation adequacy than others with a higher $\mathrm{CF}$, but whose generation is less correlated to the load. The CF is assumed as the ratio between the actual annual energy produced by a $\mu \mathrm{Gen}$ technology and the maximum value that could be generated if the $\mu \mathrm{Gen}$ is running full time at rated power.

In order to account for the mentioned variations and potential dependencies between variables, several scenarios may be defined. Each scenario represents a time frame including some daily hours of an annual period (for instance, the period between 7:30 a.m. and 9:30 a.m. of spring days), where the different variables may be assumed as independent or where a clear correlation may be established. The number of scenarios to be defined should fulfill two requisites: (a) the number of scenarios should be as low as possible, in order to avoid excessive computation times; and (b) should be enough to capture most of the dependencies between the load and the different generation variables.

So, the non-chronological MCS is performed in each scenario and the global results are obtained by aggregating the indices, as shown in Fig. 2. Therefore, each trial of the MCS process begins by sampling a scenario. Afterwards the load level (Ls) is sampled using a cumulative distribution function (CDF) suitable to represent the load behavior in the sampled scenario and the inverse transform method (Billinton and $\mathrm{Li}, 1994)$. The inverse transform method is also used to sample the CF of each $\mu \mathrm{Gen}$ technology on each scenario. Therefore, the behavior of the CF of the $\mu \mathrm{Gen}$ technologies on each scenario must be represented by an appropriate CDF. As shown in Section 3.2, the CF are fundamental parameters when assessing the contribution of the $\mu$ Gen systems to the available capacity of the system. Note that the scenarios can capture an irregular behavior of the CF. For instance, the behavior of the CF of a PV system may be well represented by a CDF of a uniform distribution in some scenarios. However, a triangular distribution may be more accurate in other scenarios, as discussed in Section 4.1.

\subsection{Detailed modeling}

The available generation capacity (CS) on each MCS trial results from the sum of the available capacity on centralized 
generators and the one of $\mu \mathrm{Gen}$ :

$C_{S}=\sum_{i=1}^{N G} F_{G i} \times C_{G i}+\sum_{j} F_{j, S} \times C_{j} \times C F_{j, S}$

where $N G$ is the number of centralized generators; $C_{G i}$ is the capacity of the generator $i ; F_{j, S}$ is the number of microgenerators of technology $j$ without failure on scenario $S, C_{j}$ is the individual capacity of microgenerators, $C F_{j, S}$ is the capacity factor of microgenerators of technology $j$ on scenario $S$; and $F_{G i}$ is given by

$F_{G i}=\left\{\begin{array}{lll}0 & \text { if } & U<F O R_{i} \\ 1 & \text { if } & U \geq F O R_{i}\end{array}\right.$

where $U$ is a random number between 0 and 1 with uniform distribution and $F O R_{i}$ is the forced outage rate of the generator $i$.

The value $F_{j, S}$ depends on the number of microgenerators $(n)$ and on its forced outage rate $(f)$, and follows a binomial distribution. For a relatively high number of microgenerators, the binomial distribution may be approximated by a normal distribution given by

$F_{j, S} \sim N(n(1-f) ; \sqrt{n(1-f) f})$

Therefore, the number of available microgenerators on each trial of the simulation process may be obtained by

$F_{\mu G}=n(1-f)+\sqrt{n(1-f) f} \times U_{n}$

where $U_{n}$ is a normal distributed random number with mean 0 and variance 1 .

The values of the $\mathrm{CF}$ of each $\mu \mathrm{Gen}$ technology on each trial of the simulation process $\left(C F_{j, S}\right)$ are sampled using the inverse transform method and suitable CDF (as explained in Section 3.1). These CDF have to be obtained taking into consideration the behavior of the CF on each defined scenario. Concerning PV generators, the $\mathrm{CF}$ values are calculated by

$C F_{P V}=\frac{I \times A \times \eta}{P_{p}}=\frac{\eta \times I}{v}$

where $P_{p}$ is the peak power of the generator; $I$ is the incident solar irradiance $\left(\mathrm{W} / \mathrm{m}^{2}\right) ; A$ the surface of the PV generator; $\eta$ the efficiency of the PV system (including the interfaces); and $v$ the ratio between the peak power and the surface of the generator $\left(\mathrm{W} / \mathrm{m}^{2}\right)$.

By using typical values for $\eta$ and $v$, the $C F$ of a typical PV generator becomes a function of the incident irradiance, which is influenced by several factors, namely: the geographical location of the PV system; the climacteric conditions; the generator orientation and inclination with the horizontal; and the annual and daily periods. Naturally, it is unpractical to define a CDF for each potential combination of those factors. Therefore, typical profiles of irradiance should be used in order to obtain the CDF that represent the CF behavior of a typical PV generator. A possible approach is to use the irradiance profile of a typical day of each month for a set of locations (assumed as appropriate to model geographical variation of the irradiance). Those typical profiles have to be constructed taking into consideration the usual installation conditions (orientation and tilt angle) of the PV panels.

Concerning micro-CHP, the CF also tends to vary along the year and along the hours of the day in accordance to the thermal energy requirements. An estimative of the CF for micro-CHP units along the time may be obtained using typical profiles of thermal energy consumption.

The behavior of the $\mathrm{CF}$ of micro-wind generators may be obtained through the use of wind velocity data and of a typical power curve of a micro-wind generator. Fig. 3 shows the typical

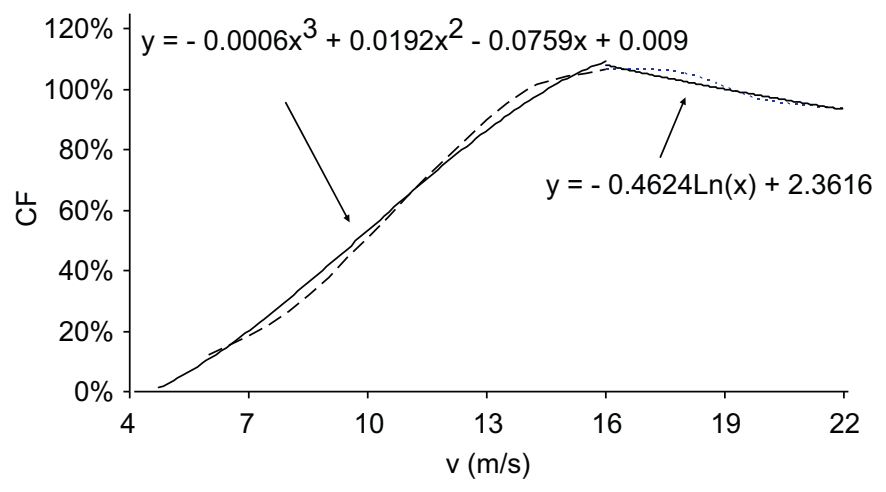

Fig. 3. Typical power curve for a micro-wind generator.

power curve used in this paper as well as its analytical approximation. By using this approximation and wind series of some geographic regions it is possible to determine series of $\mathrm{CF}$. Those series are then used to define CDF that represent the behavior of the micro-wind systems' CF on each scenario (further details are shown in Section 4).

\section{Case study}

The proposed methodology was applied to a test system designed to serve the Portuguese load data (2007 values, peak load $=9110 \mathrm{MW}$ ), but where the conventional power system was modeled assuming a set of 24 thermal power plants with a rated power of $400 \mathrm{MW}$ and $F O R=5 \%$. The initial LOLE of the system (in the absence of the $\mu \mathrm{Gen}$ ) is $27.8 \mathrm{~h}$ /year. This LOLE does not represent the typical value of the Portuguese system. However, it was used in order to obtain a sufficient range of the reliability improvements resulting from the addition of $\mu \mathrm{Gen}$ and $\mu \mathrm{Grids}$ to the system. Additional studies were made in order to assess the influence of the initial system reliability on the achieved improvements.

The CC of the $\mu \mathrm{Gen}$ and $\mu \mathrm{Grids}$ was evaluated comparing the adequacy indices of a base case without microgeneration with the indices associated to various penetrations of different technologies of microgeneration (CHP, PV and micro-wind) and the existence of $\mu$ Grids. A number of possible typical situations were analyzed, namely to conclude about the sensitivity of the results to parameters such as the reliability of the power system and the number, dimension and unavailability of the microgenerators.

\subsection{Scenarios and CDF definition}

The scenarios were defined taking into consideration the seasonal and daily behavior of the system load and of the CF of the $\mu$ Gen systems (namely concerning PV and micro-CHP technologies). To account for the seasonality the year was divided into four annual periods corresponding to the annual seasons. Afterwards, the behavior of the CF and of the system load on a typical day of each season was considered in order to define daily periods. Fig. 4 shows this information for the Portuguese situation. The PV $\mathrm{CF}$ was defined assuming $\eta=10 \%$ and $v=100 \mathrm{~W} / \mathrm{m}^{2}$. The irradiance values were obtained on PVGIS Project (2009), where information about average irradiance for periods of $15 \mathrm{~min}$ of a typical day of each month is available. Concerning the load, the chronological load diagram of the power system was used. The $\mathrm{CF}$ of micro-CHP systems was defined based on reasonable assumptions.

Based on the information of Fig. 4, six daily periods (P1-P6) were defined in order to take into account the daily behavior of 


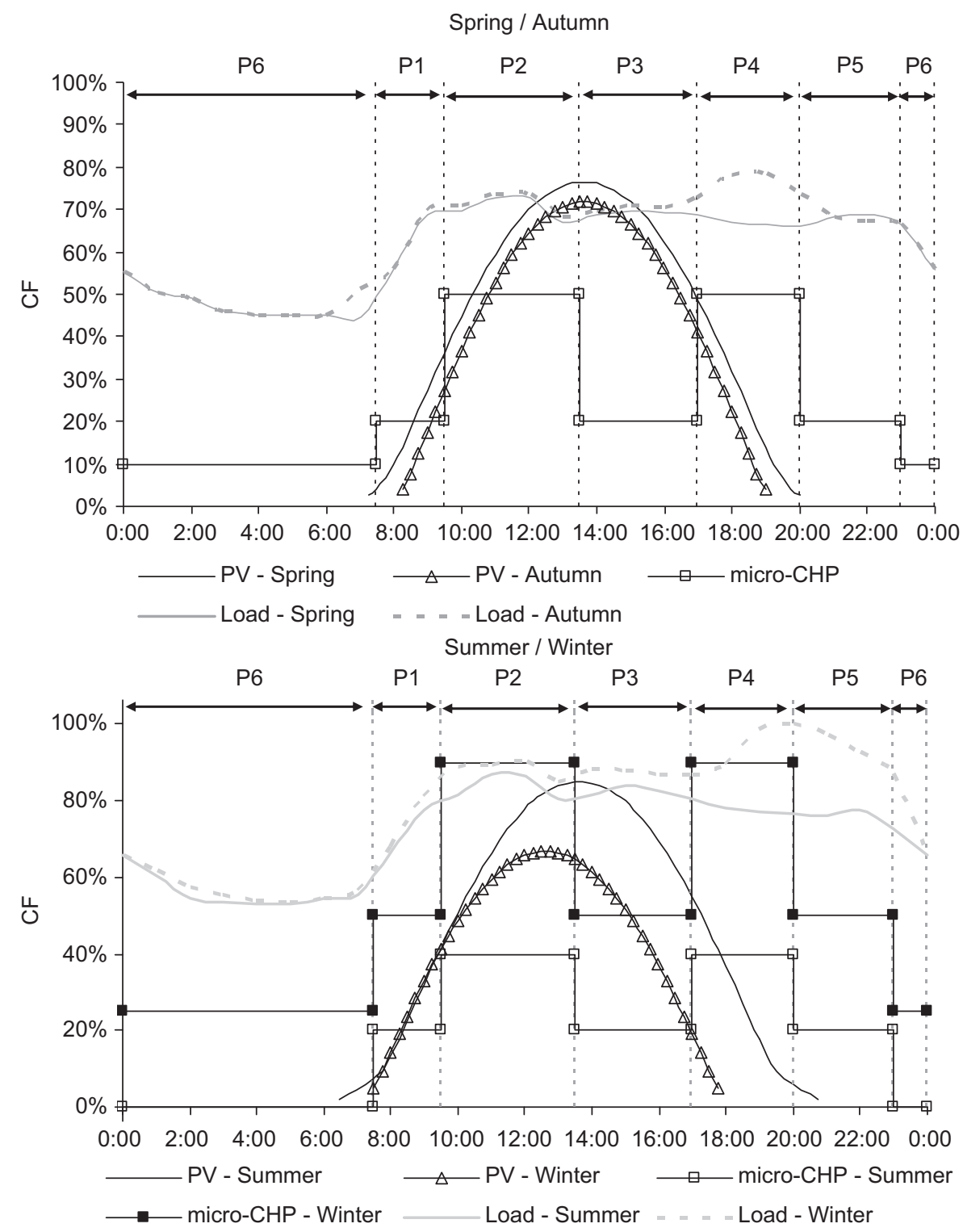

Fig. 4. Scenarios definition.

Table 1

Defined scenarios.

\begin{tabular}{|c|c|c|c|c|c|}
\hline \multicolumn{6}{|l|}{ Daily period } \\
\hline 07:30-09:30 h & 09:30-13:30 h & $13: 30-17: 00 \mathrm{~h}$ & $17: 00-20: 00 \mathrm{~h}$ & $20: 00-23: 00 \mathrm{~h}$ & $00: 00-07: 30$ h 23:00-24:00 h \\
\hline Spring-P1 & Spring-P2 & Spring-P3 & Spring-P4 & Spring-P5 & Spring-P6 \\
\hline Summer-P1 & Summer-P2 & Summer-P3 & Summer-P4 & Summer-P5 & Summer-P6 \\
\hline Autumn-P1 & Autumn-P2 & Autumn-P3 & Autumn-P4 & Autumn-P5 & Autumn-P6 \\
\hline Winter-P1 & Winter-P2 & Winter-P3 & Winter-P4 & Winter-P5 & Winter-P6 \\
\hline
\end{tabular}

the CF and load level. So, in this case, 24 scenarios were defined, corresponding to six time frames within a typical day and four annual periods (Table 1 ).

Subsequently to the scenarios' definition, the CDF that characterizes the behavior of the CF of $\mu \mathrm{Gen}$ and of the system load on each scenario were defined. Concerning the load, the CDF for each scenario was obtained using the chronological load diagram of the power system (15 min periods). Based on that information, empirical distributions of the load level at each scenario were created and then approximated by using uniform probability distribution functions (pdf). Fig. 5 shows the CDF obtained for the period "Spring P1".

Once the CDF were defined, the load level on each trial of the MCS is obtained by

$$
L_{s}=m_{s} U+b_{s}
$$


where $m_{s}$ and $b_{s}$ are the coefficients of the line of approximation in scenario $s$ and $U$ is a random number between 0 and 1 with a uniform distribution.

The CDF that represent the CF of the PV generators were obtained using data about irradiance for each of the defined scenarios. Fig. 6 shows the CDF obtained for two of the defined scenarios, specifically for the "Spring-P1" and "Summer-P1" scenarios. In this case, different probability distribution functions were used to approximate the CDF in each case, namely the triangular and the uniform distributions. Therefore, for the scenarios where a uniform distribution applies, the CF used on each trial of the MCS was sampled using a procedure similar to the one used to sample the load level. For the scenarios where a triangular distribution applies, the CF was sampled by

$C F=\left\{\begin{array}{lll}a+\sqrt{U(m-a)(b-a)} & \text { if } & 0 \leq U \leq \frac{m-a}{b-a} \\ b-\sqrt{(1-U)(b-m)(b-a)} & \text { if } & \frac{m-a}{b-a}<U \leq 1\end{array}\right.$

where $U$ is a random number between 0 and 1 with a uniform distribution, and $m, a$ and $b$ are, respectively, the mode, the lower and the upper boundary parameters of the triangular distribution.

Note that because there is a clear correlation between the system load level and the CF of the PV generators at the scenarios "Spring-P1", "Summer-P1", "Autumn-P1" and "Winter-P1" (see Fig. 4), a couple sampling was used in order to sample those variables. This means that when those scenarios are sampled, the same random number was used for sampling the load level and the CF of PV generators.

Regarding micro-wind systems, CF values were calculated taking into consideration the data about wind velocity and a typical power curve (Fig. 3). To account for the geographic variability of the wind velocity, wind data of three locations were considered (Porto, Lisbon and Faro). In this case, the concept of conditional probability was employed, concerning the situations of wind absence, so sampling was first used to decide whether or not $C F=0$. Then, if $C F>0$, a conditional $C D F$ was used to sample a value for $C F$. Fig. 7 shows for the scenario "Spring-P1" the empirical distribution and its approximation by using an

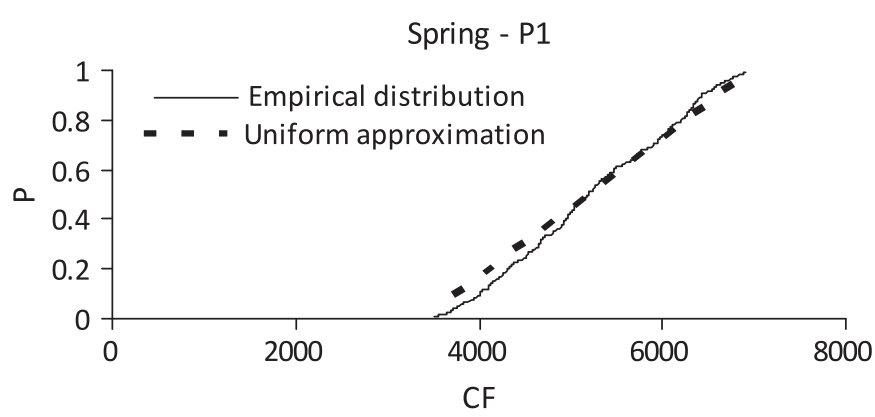

Fig. 5. CDF for load level.

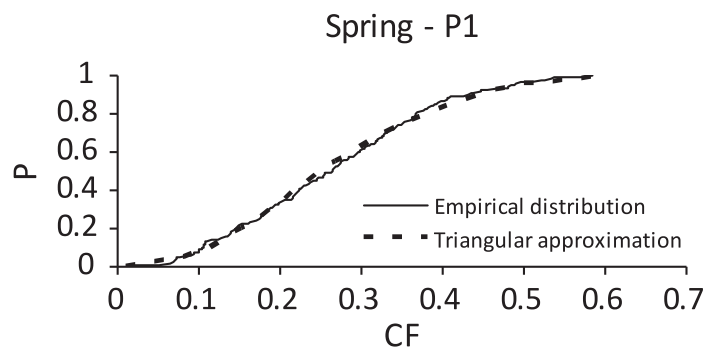

exponential $C D F$ for situations where $C F>0$. Note that, in this case, $P(C F=0)=46.2 \%$.

At such circumstances, the $C F$ to be used at each trial of the MCS is obtained by generating a random number, $U$, with uniform distribution and belonging to the interval $[0,1]$ and comparing it with the value $P(C F=0)$. The $C F$ equals 0 when $U \leq P(C F=0)$. When $U>P(C F=0)$ the conditional CDF, based on an exponential distribution, is used in order to obtain the $C F$ :

$C F_{s}^{\text {Eol }}=-\lambda_{s} \times \ln \left(U^{\prime}\right)$

where $\lambda_{s}$ is the mean of the exponential distribution corresponding to scenario $s$ and $U^{\prime}$ is a random number with uniform distribution belonging to the interval $[0,1]$.

Concerning micro-CHP systems, their greater predictability allows the use of a typical value for $C F$ of each scenario (the values depicted in Fig. 4 were used).

\subsection{Results}

The simulations have shown that different $\mu$ Gen technologies present dissimilar impacts on the system adequacy indices (mainly due to the different correlations between the generation profiles of the $\mu \mathrm{Gen}$ technologies and the load behavior). Therefore, the CC of $\mu$ Gen systems differs according to their technology. Figs. 8 and 9 show the main results, with the CC being defined as the ratio between the thermal capacity that could be removed from the system (without changing the LOLE index) and the $\mu$ Gen's total added capacity $P$.

Note that the influence of the individual capacity and the unavailability of the microgenerators on the $C C$ is limited. For instance, the CC of a set of 160,000 micro-CHP systems with $C_{j}=3 \mathrm{~kW}$ and $f=10 \%$ is $64 \%$. Therefore, the addition of $480 \mathrm{MW}$ of micro-CHP allows us to remove 307.2 MW of thermal generation, keeping the initial reliability of the system $(\mathrm{LOLE}=27.8 \mathrm{~h})$. Halving the unavailability of the generators $(f=5 \%)$ increases the $C C$ in $4.7 \%$, to a value $C C=67 \%$. If the unavailability is increased in $250 \%(f=25 \%)$ the CC decreases in $17 \%$, to a value of $53 \%$. Similar results can be obtained for the other $\mu \mathrm{Gen}$ technologies studied. Note that, as Fig. 8 shows, the influence of

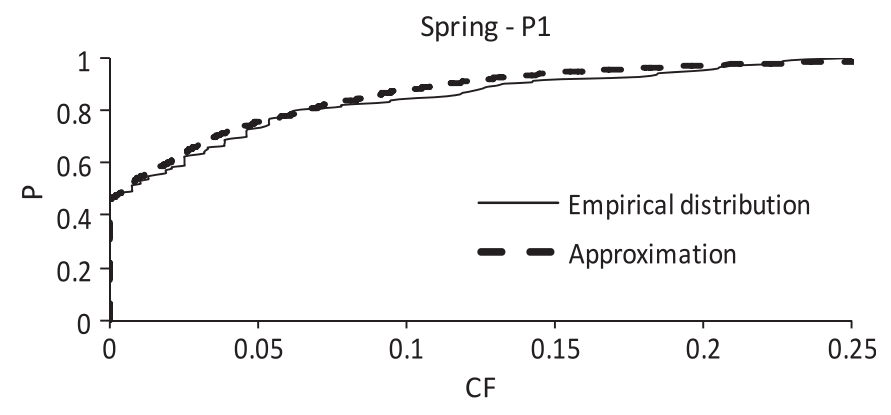

Fig. 7. CDF for micro-wind capacity factor.

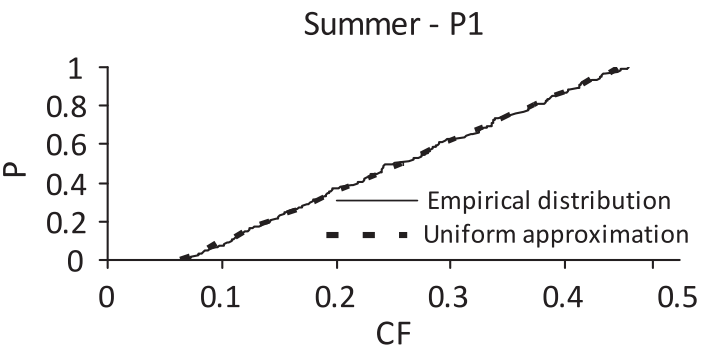

Fig. 6. CDF for PV capacity factor. 


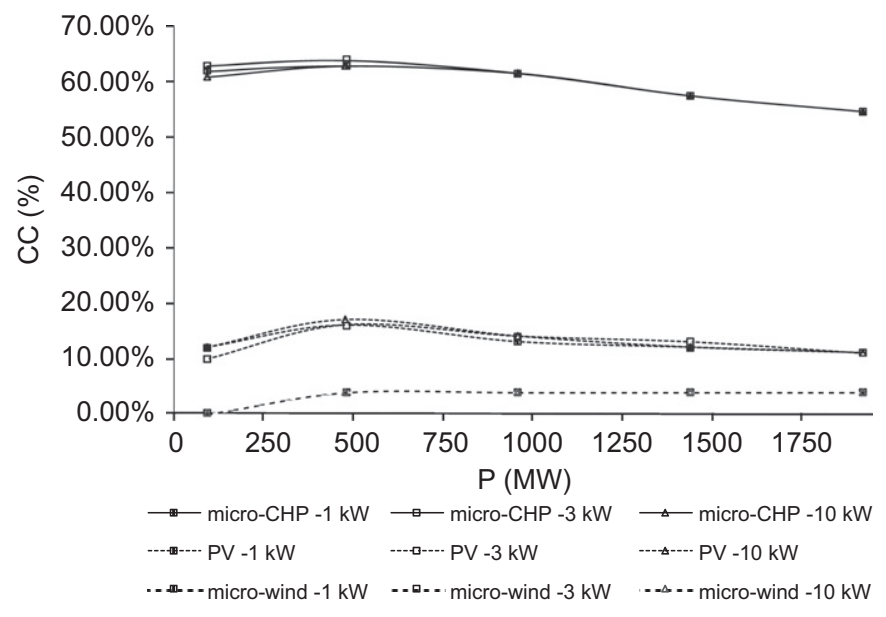

Fig. 8. Influence of the individual capacity on the CC.

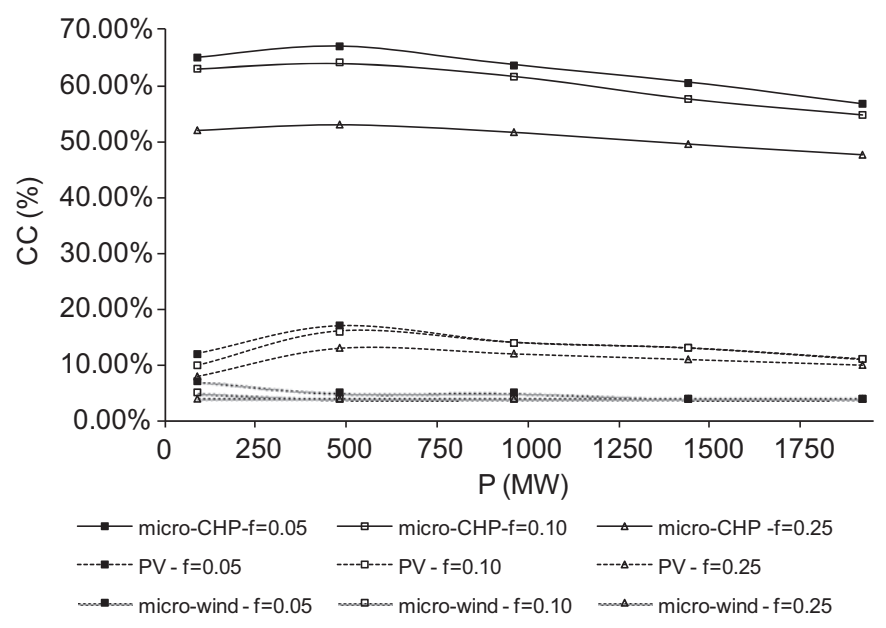

Fig. 9. Influence of FOR of $\mu \mathrm{Gen}$ on the CC.

the rated power of the microgenerators is even lower. The results of Figs. 8 and 9 also show that the variation of the $C C$ values with the penetration level is limited. This is an important feature from the regulatory point of view, since a typical value of $C C$ can be adopted for each technology regardless of the technology penetration.

In order to evaluate the influence of the initial reliability level of the power system, some tests have been made assuming different values of initial $\operatorname{LOLE}(76.7,27.8,2.28$ and 0.54 h). Fig. 10 shows the relevant results, for $\mu$ Gen systems with rated power of $3 \mathrm{~kW}$ and $f=10 \%$.

The results show that the initial reliability level of the electrical system influences the CC of the $\mu \mathrm{Gen}$ units in different ways. In fact, the CC of micro-CHP units tends to increase when the initial system reliability is higher. The opposite situation occurs for PV and micro-wind systems. The reason for this is the following: as the system reliability increases, the loss of load situations tend to be more concentrated in peak hour time periods. Once the micro-CHP generation profile has an important correlation with the system load, its contribution to avoid loss of load situations increases, and so does the CC. Concerning PV and micro-wind systems, the increase in the system reliability tends to eliminate some loss of load situations that, otherwise, would be removed when PV and micro-wind capacity are added to the system. Therefore the CC of such units tends to decrease when the
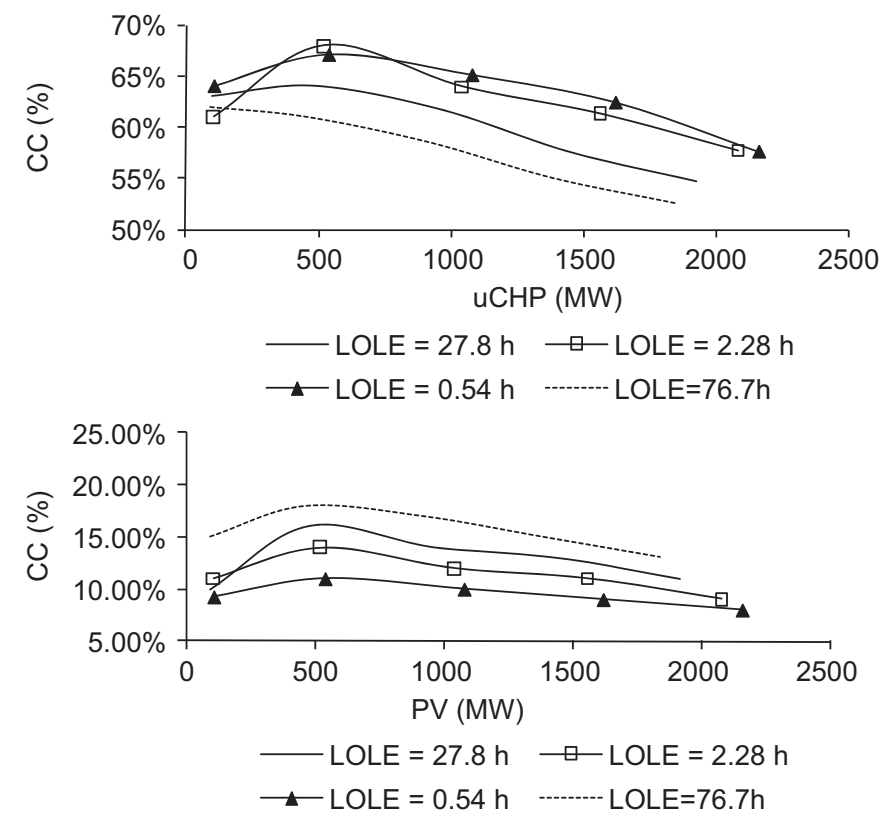

Fig. 10. Influence of initial reliability on CC.

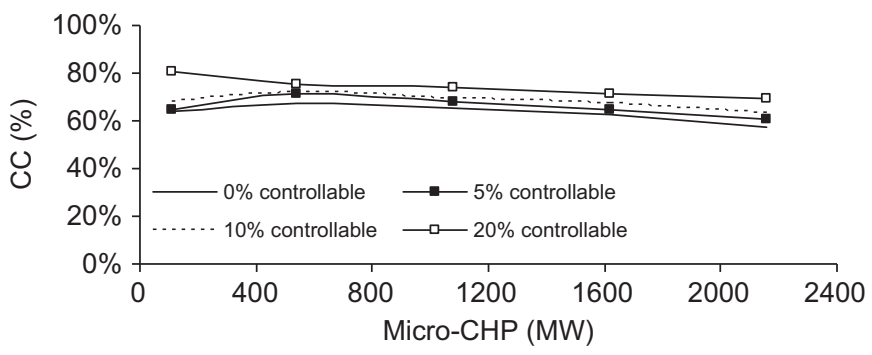

Fig. 11. Influence of $\mu \mathrm{Grid}$ on the CC of micro-CHP systems.

system reliability increases. It is important to stress that the CC of PV systems would increase if the system load peak would coincide with the time periods of greater solar irradiation. Nevertheless, the influence of the initial reliability of the system on the CC is limited in any case.

As mentioned in Section 3 the specific contribution of a $\mu \mathrm{Grid}$ to the generating adequacy depends on the controllable internal $\mu \mathrm{Gen}$ and loads. Fig. 11 shows the influence of the $\mu \mathrm{Grids}$ on the CC of micro-CHP systems when different percentages of such systems are assumed as controllable (for a system with an initial LOLE of $0.54 \mathrm{~h}$ ). Concerning load control, the CC, expressed in MW, corresponds to the total system load controllable by the $\mu$ Grid's action.

\section{Conclusions}

The concept of capacity credit was used as a measure of the contribution of $\mu \mathrm{Gen}$ and $\mu \mathrm{Grids}$ to the generation adequacy of a power system, as part of a methodology based on Monte-Carlo simulation. Potential seasonal and daily dependencies between the system load and the generation profile of the different $\mu \mathrm{Gen}$ technologies (micro-CHP, PV, micro-wind, etc.) were taken into account through the definition of scenarios that also facilitated an 
adequate modeling of the probability density functions of the capacity available.

The methodology was applied to a realistic system with the load level of Portugal. The results show that the CC of different $\mu \mathrm{Gen}$ technologies is significantly influenced by the correlation between the respective generation profiles and the system load behavior, with a clear advantage of CHP. It was also possible to corroborate the positive influence in system's reliability of multiple small units as a replacement of big units. On the other hand, the CC values are just slightly influenced by the rated power and individual unavailability of the microgenerators, whatever is the $\mu$ Gen technology.

The CC of $\mu \mathrm{Gen}$ is also influenced by the global level of reliability of the power system, with different effects for CHP (higher CC in reliable systems) and PV and wind power (higher CC in unreliable systems). However, in both cases the influence is limited.

Therefore, it seems possible to use typical values for parameters like rated power, individual unavailability and the global level of reliability, without incurring in significant errors when estimating the $\mathrm{CC}$ of $\mu \mathrm{Gen}$ and $\mu$ Grids. This feature is especially interesting for remuneration design and other regulatory purposes.

\section{References}

Amelin, M., 2009. Comparison of capacity credit calculation methods for conventional power plants and wind power. IEEE Transactions on Power Systems 24 (2), 685-691.

Bae, I.S., Kim, J.O., 2007. Reliability evaluation of distributed generation based on operation mode. IEEE Transactions on Power Systems 22 (2), 785-790.

Billinton, R., Li, W., 1994. Reliability Assessment of Electric Power Systems Using Monte Carlo Methods. Plenum Press, New York.

Caire, R., Retiere, N., Martino, S., Andrieu, C., Hadjsaid, N., 2002. Impact assessment of LV distributed generation on MV distribution network. In: IEEE Power Eng. Society Summer Meeting, vol. 3. pp. 1423-1428.
Costa, P.M., Matos, M.A., Lopes, J.A.P., 2008. Regulation of microgeneration and microgrids. Energy Policy 36 (10), 3893-3904.

Costa, P.M., Matos, M.A., 2009. Assessing the contribution of microgrids to the reliability of distribution networks. Electric Power Systems Research 79 (2), 382-389.

Deutsche Energie-Agentur GmbH, 2005. Planning of the grid integration of wind energy in Germany onshore and offshore up to year 2020 (dena grid study) (summary in German and English available on-line from : 〈http://www.dena. de/de/themen/thema-reg/projekte/projekt/netzstudie-i/ $\rangle$.

DTI, 2004. System integration of additional micro-generation (available on-line as: $\langle$ http://www.berr.gov.uk/files/file15192.pdf 〉.

Garver, L.L., 1966. Effective load carrying capability of generating units. IEEE Transactions on Power Apparatus and Systems PAS-85 (8), 910-919.

Hawkes, A.D., Leach, M.A., 2008. The capacity credit of micro-combined heat and power. Energy Policy 36 (4), 1457-1469.

He Yau, P. 2006. Generation system reliability evaluations with intermittent renewable. Master Thesis. University of Strathclyde (available on-line as 〈http://www.esru.strath.ac.uk/Documents/MSc_2006/phoon.pdf 〉).

Lasseter, R.H., Piagi P., 2004. Microgrid: a conceptual solution, PESC-04, Germany.

Luickx, P., Vandamme, W., Pérez, P.S., Driesen, J., D’haeseleer, W., 2009. Applying Markov chains for the determination of the capacity credit of wind power. In: 6th International Conference on the European Energy Market, Leuven, Belgium, May 27-29, 2009. 〈http://www.mech.kuleuven.be/energy/re sources/docs/papers/pdf/WP\%20EN2009-004.pdf $\rangle$.

Milligan, M., Porter, K., 2006. The capacity value of wind in the United States: methods and implementation. The Electricity Journal 19 (2), 91-99 (Elsevier).

Milligan, M., 2000. Modelling utility-scale wind power plants. Part 2: capacity credit. Wind Energy 3 (4), 167-206. doi:10.1002/we.36.

Project $\mu$ grids, 2008. 〈http://www.microgrids.eu/default.php $\rangle$.

Pudaruth, G.R., Li, F., 2009. Locational capacity credit evaluation. IEEE Transactions on Power Systems 24 (2), 1072-1079.

Pudaruth, G.R., Li, F., 2008. Capacity credit evaluation: a literature review. In Electric utility deregulation and restructuring and power technologies, DRPT 2008, pp. 2719-2724.

Pudjianto, D., Strbac, G., Overbeeke, F., Androutsos, A.I., Larrabe, Z., Saraiva, J.T., 2005. Investigation of regulatory, commercial, economic and environmental issues in microgrids. In: International Conference on Future Power Systems.

PVGIS Project, 2009. 〈http://re.jrc.ec.europa.eu/pvgis/ 〉.

Saraiva, J.T., Lopes, J.P., Hatziargyriou, N., Jenkins, N., 2003. Management of microgrids. In: International Electrical Equipment Conference, Bilbao, October, 2003 (presentation available online from: 〈http://www.microgrids.eu/mi cro2000/index.php? page=publications $>$ ). 